\title{
Flavor altering excitations of composite fermions
}

\author{
Michael R. Peterson and Jainendra K. Jain \\ Department of Physics, 104 Davey Laboratory, The Pennsylvania State University, University Park, Pennsylvania 16802
}

(Dated: November 15, 2018)

\begin{abstract}
Past theoretical studies have considered excitations of a given flavor of composite fermions across composite-fermion quasi-Landau levels. We show that in general there exists a ladder of flavor changing excitations in which composite fermions shed none, some, or all of their vortices. The lowest energy excitations are obtained when the composite fermions do not change their flavor, whereas in the highest energy excitations they are stripped of all of their vortices, emerging as electrons in the final state. The results are relevant to the intriguing experimental discovery [1] of Hirjibehedin et al. of coexisting excitation modes of composite fermions of different flavor in the filling factor range $1 / 3>\nu \geq 1 / 5$.
\end{abstract}

PACS numbers: PACS: 71.10.Pm,73.43.-f

The quantum fluid of interacting electrons in the lowest Landau level, which manifests itself most dramatically through the fractional quantum Hall effect [2], is characterized by the formation of a new class of fermions called composite fermions 3] (CFs). The composite fermion is the bound state of an electron and an even number $(2 p)$ of quantized vortices, denoted by ${ }^{2 p} \mathrm{CF}$. Composite fermions of different flavors (that is, with different numbers of attached vortices) occur in different filling factor regions, where the filling factor, $\nu=\rho h c / e B$ is the number of occupied Landau levels (LLs). (Here $\rho$ is the two-dimensional density of electrons and $B$ is the external magnetic field.) Specifically, ${ }^{2 p} \mathrm{CFs}$ are relevant in the range $\frac{1}{2 p-1}>\nu \geq \frac{1}{2 p+1}$.

Light scattering has proved to be a powerful tool for investigating the nature of excitations of this quantum fluid [4, 5, 6, 7]. The present work has been motivated by the recent light scattering experiment by Hirjibehedin et al. [1], which investigated the filling factor range $1 / 3 \geq \nu \geq 1 / 5$, where the relevant composite fermions carry four vortices $\left({ }^{4} \mathrm{CFs}\right)$. A remarkable aspect of the experimental results is that while new low-energy excitations appear for $\nu<1 / 3$, as expected, the old excitations from $\nu>1 / 3$ do not disappear but rather evolve continuously as the filling factor changes from $\nu>1 / 3$ to $\nu<1 / 3$; in other words, there is a coexistence of excitations of both ${ }^{4} \mathrm{CFs}$ and ${ }^{2} \mathrm{CFs}$ in the low filling factor region.

We begin by presenting a physical explanation. A consideration of the filling factor region $\nu>1 / 3$ (Fig. 1) provides valuable insight into this intriguing observation. Here, we have the lowest electronic LL partially occupied, and, as a result of the repulsive interaction, electrons transform into ${ }^{2} \mathrm{CFs}$; the lowest electronic LL thus splits into quasi-Landau levels [8] (QLLs) of ${ }^{2} \mathrm{CFs}$ (middle column of Fig. 1). Now, the intra-LL excitations of electrons are described as inter-QLL excitations of ${ }^{2} \mathrm{CFs}$. However, the system obviously still supports the interelectronic LL excitation, namely the Kohn mode. We see in this simple case the coexistence of excitation modes

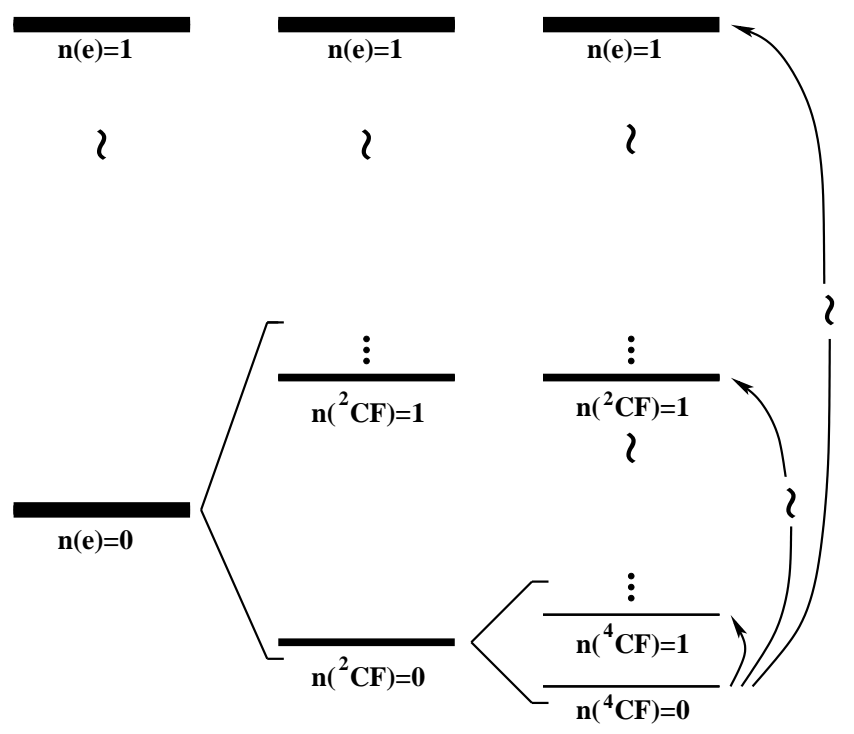

FIG. 1: Schematic view of how the lowest electronic Landau level splits into quasi-Landau levels of composite fermions carrying two vortices $\left({ }^{2} \mathrm{CFs}\right)$, and the lowest ${ }^{2} \mathrm{CF}$ quasi-Landau level further splits into ${ }^{4} \mathrm{CF}$ quasi-Landau levels. The (quasi)Landau level index is denoted by $\mathrm{n}(\mathrm{P})$ where "P" is the type of relevant particle, namely "e" (electron), "2 CF," or "4 CF." On the rightmost column, the three ladders of flavor changing excitations are indicated. The lowest rung in each ladder corresponds to: $\Delta^{4 \rightarrow 4}$ from $\mathrm{n}\left({ }^{4} \mathrm{CF}\right)=0$ to $\mathrm{n}\left({ }^{4} \mathrm{CF}\right)=1 ; \Delta^{4 \rightarrow 2}$ from $\mathrm{n}\left({ }^{4} \mathrm{CF}\right)=0$ to $\mathrm{n}\left({ }^{2} \mathrm{CF}\right)=1 ; \Delta^{4 \rightarrow 0}$ from $\mathrm{n}\left({ }^{4} \mathrm{CF}\right)=0$ to $\mathrm{n}(\mathrm{e})=1$.

at two different energy scales: the low energy $\Delta^{2 \rightarrow 2}(k)$ excitations conserve the $\mathrm{CF}$ flavor, whereas the high energy $\Delta^{2 \rightarrow 0}(k)$ excitations involve transformation of ${ }^{2} \mathrm{CF}$ into ${ }^{0} \mathrm{CF}$ (electron). In the former case, the composite fermions maintain their integrity during the transition, whereas in the latter case they leave their vortices behind in the lowest electronic Landau level, thus converting into excited electrons in the second level.

For $\nu<1 / 3$ the situation is analogous. The lowest ${ }^{2} \mathrm{CF}$ QLL undergoes a further splitting into QLLs of ${ }^{4} \mathrm{CFs}$, as 


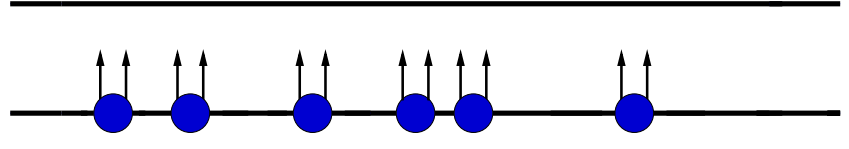

(a)

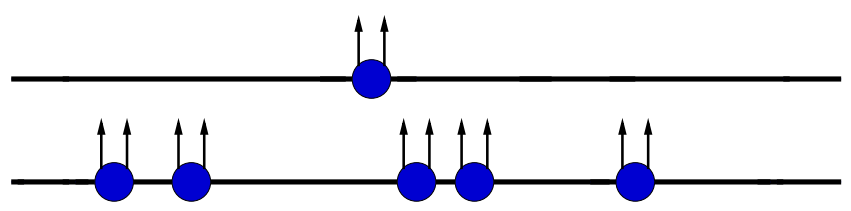

(b)

FIG. 2: The ${ }^{2} \mathrm{CF}$ basis states included in our study to investigate the excitations $\Delta^{4 \rightarrow 4}$ and $\Delta^{4 \rightarrow 2}$ in the filling factor range $1 / 3>\nu \geq 1 / 5$. (The ${ }^{2}$ CFs are shown as electrons carrying two arrows, and the horizontal lines depict their quasi-Landau levels.) These are constructed from the analogous basis states for electrons. Keeping only states of the type given in (a) produces the $\Delta^{4 \rightarrow 4}$ excitations shown by empty circles in Fig 3

shown in the rightmost column of Fig. 11 Although we have only ${ }^{4} \mathrm{CFs}$ in the ground state, there are three ladders of excitation: (i) $\Delta^{4 \rightarrow 4}(k)$ excitations, which were not present for $\nu>1 / 3$; (ii) $\Delta^{4 \rightarrow 2}(k)$ excitations involving a change of ${ }^{4} \mathrm{CFs}$ into ${ }^{2} \mathrm{CFs}$, which are a continuous evolution of $\Delta^{2 \rightarrow 2}(k)$ excitations for $\nu>1 / 3$; and (iii) $\Delta^{4 \rightarrow 0}(k)$, which are similarly close cousins of $\Delta^{2 \rightarrow 0}(k)$ and $\Delta^{0 \rightarrow 0}(k)$. This is our physical explanation for the experimental result of Ref. 1 showing a coexistence of several kinds of excitations.

To test these ideas quantitatively through a microscopic calculation, we need to construct a model that can deal with both $\Delta^{4 \rightarrow 4}$ and $\Delta^{4 \rightarrow 2}$ excitations simultaneously. The key observation that makes this possible is that the intra-QLL excitations of a given flavor of composite fermions are the inter-QLL excitations of higher order composite fermions. For example, at filling factors of the type $\nu=n /(2 n+1)$ (with $n$ integer), which are described in terms of ${ }^{2} \mathrm{CFs}$, the intra-LL excitations of electrons $\left({ }^{0} \mathrm{CFs}\right)$ are inter-QLL excitations of ${ }^{2} \mathrm{CFs}$. If we diagonalize this problem within the lowest electronic LL, we will miss the Kohn mode. In order to keep this mode, we must expand our basis space to allow at least one electron to occupy the second electronic LL; then we get not only all the $\Delta^{2 \rightarrow 2}$ excitations, but also the lowest energy $\Delta^{2 \rightarrow 0}$ excitations. This idea can be transported straight- forwardly to the filling factor region $1 / 3>\nu \geq 1 / 5$ by the vortex attachment trick of the $\mathrm{CF}$ theory, which relates $\nu^{*}$ to $\nu=\nu^{*} /\left(2 \nu^{*}+1\right)$. We first construct an electron basis at $\nu^{*}$, wherein we keep all basis states in which either all electrons are in the lowest LL or one electron occupies the second LL. By attaching two vortices to each particle, we generate from it, following methods outlined below, a ${ }^{2} \mathrm{CF}$ basis at $\nu$ shown schematically in Figs. 2 and 2 b. We then diagonalize the interaction Hamiltonian in this basis. An explicit calculation for $N=5$ and 6 for certain filling factors in the range $1 / 3>\nu \geq 1 / 5$ gives the spectrum in Fig. 3] shown by filled circles.

In order to identify the states at $\nu$ that correspond to the Kohn mode at $\nu^{*}$, we repeat the above calculation without the Kohn mode (that is, by working strictly within the lowest LL at $\nu^{*}$; Fig. 2a). We find that this gives a subset of the previous states, shown by empty circles in Fig. 3. (All empty circles sit on filled circles; for a given eigenstate, the energy of the filled circle is slightly lower than that of the empty circle because of the greater variational freedom in the expanded basis, but the difference is so small in most cases that the empty circles fully cover the filled ones.) The states not covered by empty circles are therefore inter-QLL excitations of ${ }^{2} \mathrm{CFs}$, that is, $\Delta^{4 \rightarrow 2}$ excitations.

In short, our approach is to model the $1 / 3>\nu \geq 1 / 5$ region in terms of ${ }^{2} \mathrm{CFs}$ - as opposed to ${ }^{4} \mathrm{CFs}$, which would be the natural choice if we were only interested in the lowest energy excitations. Then, the intra-QLL excitations of ${ }^{2} \mathrm{CFs}$ give us the inter-QLL excitations of ${ }^{4} \mathrm{CFs}$, namely $\Delta^{4 \rightarrow 4}$; at the same time the inter-QLL excitations of ${ }^{2} \mathrm{CFs}$ correspond to the $\Delta^{4 \rightarrow 2}$ excitations.

There is a remarkable separation of energy scales in Fig. 3 between the inter- and intra-QLL excitations of ${ }^{2} \mathrm{CFs}$, which is entirely consistent with the physics described above. A similar effect exists for the inter- and intra-LL excitations of electrons, which are governed by different energies, namely the cyclotron energy and the interaction energy. For ${ }^{2} \mathrm{CFs}$, however, both the interand intra-QLL energies are determined by the interaction energy, and the emergence of two energy scales is not obvious a priori. Fig. 3 also shows exact diagonalization results (dashes). There is an excellent agreement between the dashes and the circles, further confirming the validity of our picture. In particular, the exact spectra also show two distinct energy scales. (In fact, once we understand the physics, we can identify the $4 \rightarrow 4$ and $4 \rightarrow 2$ energies directly from the exact spectrum, which is what we do below.) Still higher energy states can be obtained within the framework of the CF theory by exciting more than one composite fermion to higher CF quasi-Landau levels, but our interest here is in the lowest excitation at each flavor changing ladder.

We next compare our results with experiment. In an ideal situation, inelastic light scattering only probes the excitations close to zero wave vector, but slight disorder, 


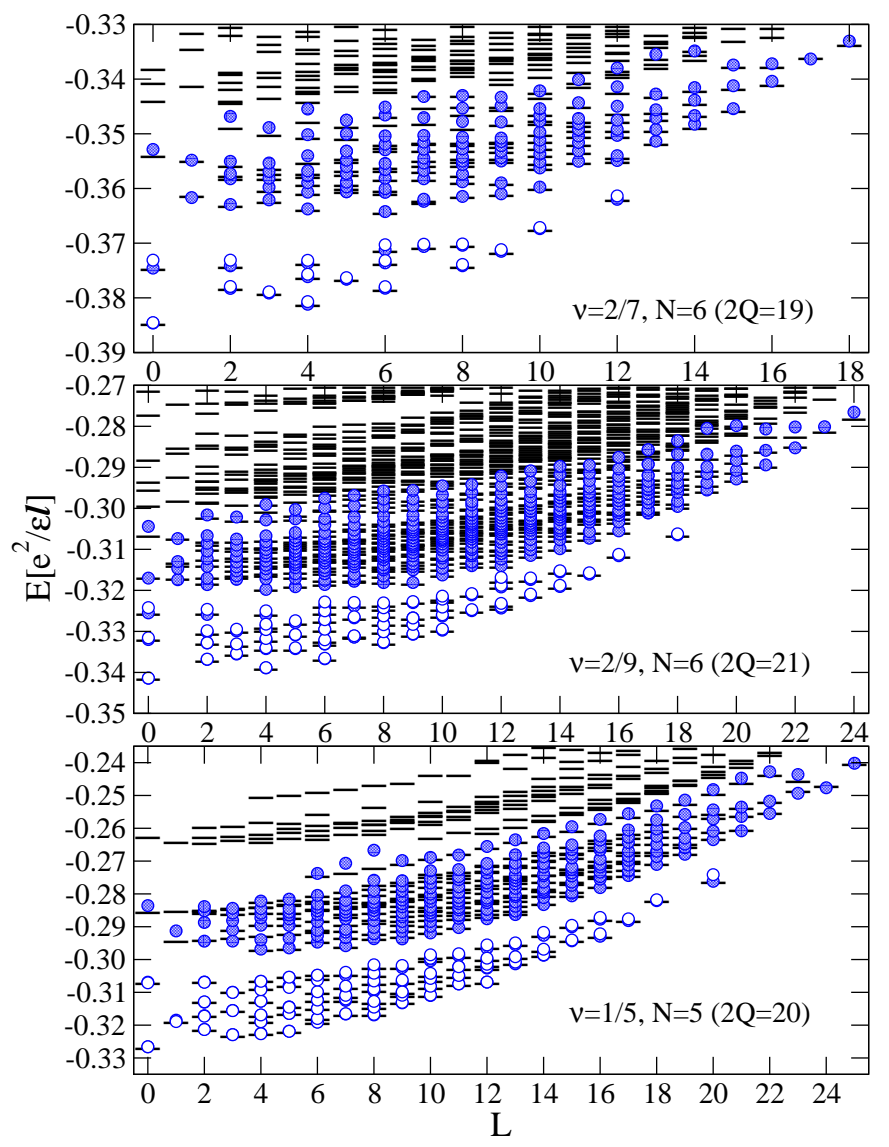

FIG. 3: Numerical results are shown for filling factors $\nu=2 / 7,2 / 9$, and $1 / 5$ for $N=6,6$, and 5 particles, respectively. The spherical geometry is used in the calculation, with the magnetic flux through the surface equal to $2 Q h c / e$. The filled circles show the spectrum calculated from the CF theory, obtained, as explained in the text, by diagonalization in the ${ }^{2} \mathrm{CF}$ basis shown schematically in Fig. 2] The empty circles are the CF states obtained when only the basis states of the type given in Fig. 27 are kept. Note that there is a filled circle underneath each empty circle (sometimes partly visible). Dashes show the exact spectrum. $L$ is the total orbital angular momentum, and the energy is reported in units of $e^{2} / \epsilon l$ where $\epsilon$ is the dielectric constant of the host semiconductor and $l=\sqrt{\hbar c / e B}$ is the magnetic length. The Monte Carlo uncertainty is smaller than the symbol size, hence not shown explicitly. The energies have been corrected for the finite size deviation in the electron density through a multiplicative factor $\sqrt{\rho / \rho_{N}}=\sqrt{2 Q \nu / N}$.

which breaks wave vector conservation, has been found to expose energies at critical points (extrema) in the $\mathrm{CF}$ exciton dispersion [4, 5, 6]. In particular, the zero wave vector mode, the minimum energy neutral excitation (roton), and the excitation that contains a far separated particle hole pair of composite fermions (which gives the transport gap measured in experiments) have been observed in Raman experiments. We will concentrate on the first two, which will be labeled $\Delta(0)$ and $\Delta(R)$, re- spectively. The transport gap is roughly the same as $\Delta(R)$ for our small systems.

The lowest energy intra- and inter- ${ }^{2} \mathrm{CF}$ QLL excitations can be identified straightforwardly from the spectra of Fig. 3 The upper panel of Fig. 4 shows the energies $\Delta^{4 \rightarrow 4}(0), \Delta^{4 \rightarrow 4}(R)$, and their $4 \rightarrow 2$ counterparts. While the $4 \rightarrow 2$ modes evolve continuously out of the $2 \rightarrow 2$ modes at $\nu \geq 1 / 3$, the $4 \rightarrow 4$ modes are new. The qualitative behavior is in agreement with experiment. There are, however, several difficulties in making a quantitative comparison with experiment.

First, the calculations are performed for a strictly two dimensional system, whereas the experimental sample is a square quantum well of width $33 \mathrm{~nm}$. We incorporate finite thickness corrections into our calculation through a self consistent local density approximation [9]. The lower panel of Fig. 4depicts the various energies after including modification in the effective two-dimensional interaction due to finite extent of the electron wave function in the transverse direction.

The other problems are more severe. Strictly speaking, one must calculate thermodynamic limits before comparing theoretical calculations to experiment. In the past, energy gaps have been found to vary substantially as a function of $N$. (For example, the transport gap at $\nu=2 / 5$ is reduced from 0.13 for $N=4$ to 0.058 in the thermodynamic limit [10].) However, because of the rather large dimension of the $\mathrm{CF}$ basis, we are not able to go to systems bigger than $N=6$ in a numerically stable manner. The calculation also neglects disorder, which is often a source of significant quantitative correction.

Next we compare our theoretical results for the roton energy with those in the experiment of Ref. 1 for the filling factor range $1 / 3 \geq \nu \geq 1 / 5$. For $\Delta^{4 \rightarrow 4}(R)$ the calculated values are approximately $\sim 0.2 \mathrm{meV}$ in this range (taking parameters of the experiment in Ref. 1), decreasing slightly with increasing magnetic field. The experimental values start at $\sim 0.2 \mathrm{meV}$ and decrease with magnetic field to $\sim 0.11 \mathrm{meV}$ at $\nu=1 / 5$. For $\Delta^{4 \rightarrow 2}(R)$ mode the theoretical value starts at approximately $\sim 0.6$ $\mathrm{meV}$ and increases with magnetic field to $\sim 2.0 \mathrm{meV}$. The experimental value starts at $\sim 0.45 \mathrm{meV}$ and goes on increasing with magnetic field to $\sim 0.7 \mathrm{meV}$. The theory is in qualitative agreement with experiment, both in regard to (i) the continuity of some modes across $\nu=$ $1 / 3$ and appearance of new modes for $\nu<1 / 3$, and (ii) the observed trends as a function of the filling factor. In light of the rather small size of the numerical system, we find the level of quantitative agreement to be satisfactory.

Finally, we briefly discuss the calculational method. It involves many steps, which will only be outlined here for lack of space; more details can be found in the literature 10, 11, 12, 13. . We have used the standard spherical geometry [14, 15] which considers $N$ electrons on the surface of a sphere with a magnetic monopole of strength $Q$ placed at the center producing a radial 


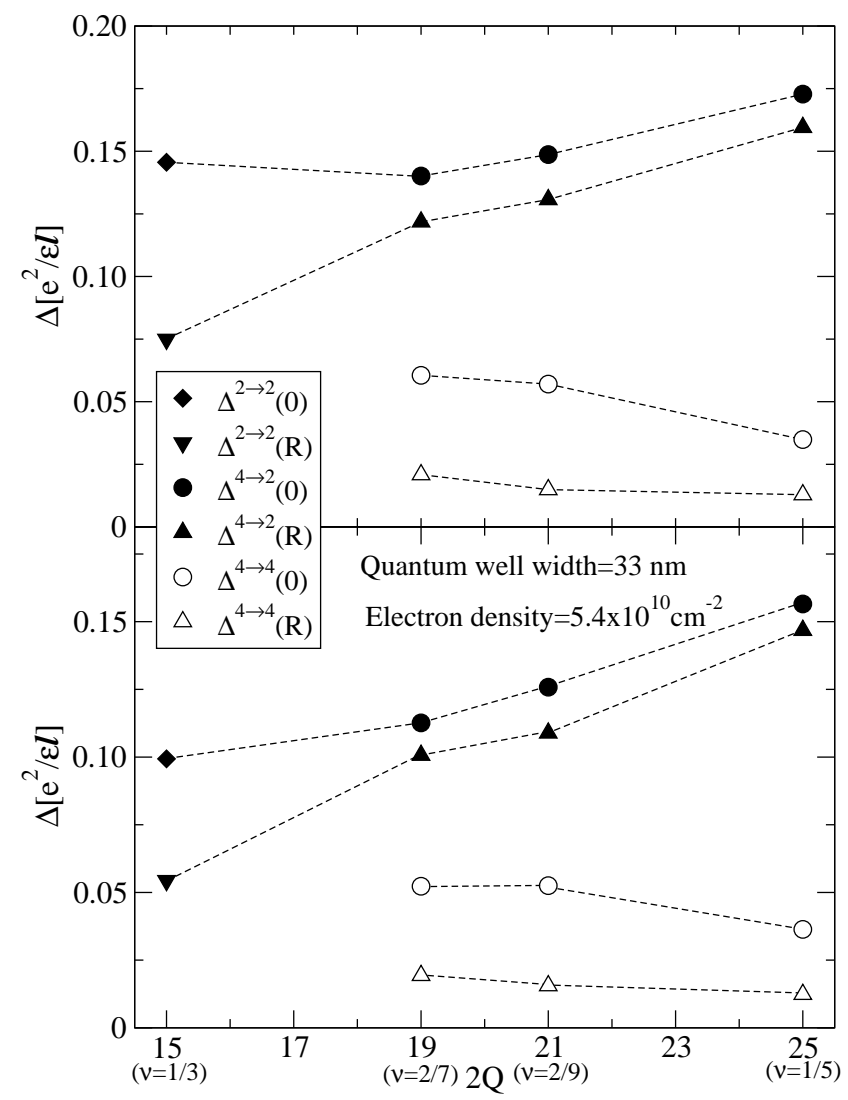

FIG. 4: Excitation energies $\Delta^{2 p \rightarrow 2 p^{\prime}}$ of the zero wave vector and roton modes are shown as a function of $2 Q$ (or magnetic field). The filling factor corresponding to a particular magnetic field strength is given in parenthesis along the horizontal axis. The upper panel gives the energies for zero thickness, whereas the lower panel incorporates corrections due to finite thickness using parameters appropriate for the experimental sample of Ref. 1 (quantum well width $=33 \mathrm{~nm}$ and density $\left.=5.4 \times 10^{11} \mathrm{~cm}^{-2}\right)$. All results are for $N=6$ particles, and the dashed lines are a guide to the eye.

magnetic field. The magnetic flux through the sphere is $2 Q \phi_{0}\left(\phi_{0}=h c / e\right.$ is called the magnetic flux quantum); $2 Q$ is an integer due to Dirac's quantization condition. The single particle eigenstates are the monopole harmonics 15] $Y_{Q n m}\left(\Omega_{j}\right)$ where $n=0,1, \ldots$ is the LL in$\operatorname{dex}, m=-(Q+n),-(Q+n)+1, \ldots,(Q+n)$ labels the $2(Q+n)+1$ degenerate states in the $n$th LL, and $\Omega_{j}$ represents the location on the sphere of electron $j$ with the usual coordinates. Since the degeneracy in successive LLs is not equal in this geometry, the ratio $N / 2 Q$ is not exactly equal to the filling factor for finite systems; the filling factor is defined as $\nu=\lim _{N \rightarrow \infty} N / 2 Q$.

According to the CF theory, $N$ strongly interacting electrons at monopole strength $Q$ are transformed into $N$ weakly interacting composite fermions at monopole strength $Q^{*}=Q-p(N-1)$. For a given $Q$ in the range $1 / 3>\nu \geq 1 / 5$, we first consider $Q^{*}=Q-N+1$, which lies in the range $1>\nu \geq 1 / 3$. We diagonalize the electron problem at $Q^{*}$ (allowing at most one electron in the second LL) to obtain eigenstates with definite total orbital angular momentum $L$. Denoting this basis by $\left\{\Phi_{Q^{*}}\right\}$, we construct a basis at $Q$ given by $\left\{P_{L L L} \Phi_{1}^{2} \Phi_{Q^{*}}\right\}$, where $\Phi_{1}$ is the wave function of one filled Landau level. $P_{L L L}$ denotes projection into the lowest electronic LL, which can be accomplished using the methods discussed previously [10]. The basis at $Q$ obtained in this manner is not orthogonal. Following the techniques in Ref. 11, we use the Gram-Schmidt procedure to obtain an orthogonal basis, and then diagonalize the interaction Hamiltonian in this basis. The various projections and Hamiltonian matrix elements require evaluation of multi-dimensional integrals, which is accomplished numerically by Monte Carlo. The uncertainty is determined by the standard deviation in the eigenvalues over different Monte Carlo runs.

A technical point in regard to our treatment of finite width should be noted. Following Ref. 16, we determine the interaction pseudopotentials 14 in the infinite planar geometry, and feed them into exact calculations in the spherical geometry. For an infinite system, this of course gives the same result as doing the calculation entirely within the spherical geometry, but for finite systems the two methods are different. For zero thickness, the use of planar pseudopotentials, as opposed to the spherical pseudopotentials, makes only a slight deviation (within $6 \%$ for our calculations) in the excitation energies; we expect that the method works equally well for finite width systems as well.

We have only considered in this work filling factors where the ground state is incompressible. It would be of interest to investigate the filling factor dependence of the various excitations energies.

This work was supported in part by the National Science Foundation under grants no. DGE-9987589 (IGERT) and DMR-0240458. We are grateful to the High Performance Computing (HPC) Group led by V. Agarwala, J. Holmes, and J. Nucciarone, at the Penn State University ASET (Academic Services and Emerging Technologies) for assistance and computing time with the LION-XL cluster.

[1] C. F. Hirjibehedin, A. Pinczuk, B. S. Dennis, L. N. Pfeiffer, and K. W. West, cond-mat/0306152 (2003).

[2] D. C. Tsui, H. L. Stormer, and A. C. Gossard, Phys. Rev. Lett. 48, 1559 (1982).

[3] J. K. Jain, Phys. Rev. Lett. 63, 199 (1989); Phys. Rev. B 417653 (1990).

[4] A. Pinczuk, B.S. Dennis, L.N. Pfeiffer, and K. West, Phys. Rev. Lett. 70, 3983 (1993).

[5] M. Kang, A. Pinczuk, B.S. Dennis, L.N. Pfeiffer, and K. West, Phys. Rev. Lett. 86, 2637 (2001); M. Kang, A. 
Pinczuk, B. S. Dennis, M. A. Eriksson, L. N. Pfeiffer, and K. W. West Phys. Rev. Lett. 84, 546 (2000).

[6] I. Dujovne, A. Pinczuk, M. Kang, B.S. Dennis, L.N. Pfeiffer, and K. West, Phys. Rev. Lett. 90, 036803 (2003).

[7] H.D.M. Davies, J.C. Harris, J.F. Ryan, and A.J. Turberfield., Phys. Rev. Lett. 78, 4095 (1997).

[8] The quasi-Landau-like levels of composite fermions must not be confused with the ordinary Landau levels of electrons.

[9] M. W. Ortalano, S. He, and S. Das Sarma, Phys. Rev. B 55, 7702 (1997); K. Park, N. Meskini, and J. K. Jain, J. Phys. Cond. Mat. 11, 7283 (1999).
[10] J.K. Jain and R.K. Kamilla, Int. J. Mod. Phys. B 11, 2621 (1997); Phys. Rev. B 55, R4895 (1997).

[11] S.S. Mandal and J. K. Jain, Phys. Rev. B 66, 155302 (2002).

[12] M.R. peterson and J.K. Jain, unpublished.

[13] S. S. Mandal, M. R. Peterson, and J. K. Jain, Phys. Rev. Lett. 90, 106403 (2003).

[14] F. D. M. Haldane, Phys. Rev. Lett. 51, 605 (1983)

[15] T. T. Wu and C. N. Yang, Nucl. Phys. B 107, 365 (1976).

[16] S. He, F. C. Zhang, X. C. Xie, and S. Das Sarma, Phys. Rev. B 42, 11376 (1990). 